\title{
Avaliação da infiltração marginal de materiais restauradores adesivos em dentes decíduos ${ }^{\dagger}$
}

\section{Evaluation of the microleakage of restorative materials in deciduous teeth}

\author{
Carlos Eduardo CARRARA* \\ Ruy César Camargo ABDO** \\ Salete Moura Bonifácio da SILVA**
}

\begin{abstract}
CARRARA, C. E.; ABDO, R. C. C.; SILVA, S. M. B. da. Avaliação da infiltração marginal de materiais restauradores adesivos em dentes decíduos. Pesqui Odontol Bras, v. 15, n. 2, p. 151-156, abr./jun. 2001.

Analisou-se a infiltração marginal nas paredes cervical e oclusal de cavidades classe V, preparadas na face vestibular de 40 caninos decíduos extraídos e restauradas com os materiais Chelon-Fil, Vitremer, Compoglass-F e Z100. Compararam-se ainda dois métodos de avaliação da penetração do corante no teste de infiltração: escore e medida linear até a parede da câmara pulpar. Os espécimes passaram por ciclagem térmica de 500 ciclos nas temperaturas de 5 e $55^{\circ} \mathrm{C}$, foram imersos em solução de fucsina básica a $0,5 \%$ por 24 horas e seccionados para visualização da infiltração marginal nas paredes cervical e oclusal. Observou-se que o Chelon-Fil apresentou maior grau de infiltração que os demais materiais. Vitremer, Compoglass-F e Z100 apresentaram infiltração semelhante. O Chelon-Fil e o Vitremer apresentaram infiltração maior na parede cervical que na oclusal, fenômeno este que não ocorreu com o Compoglass-F e a Z100, que apresentaram infiltração semelhante em ambas as paredes. Não houve diferença entre os métodos de avaliação da penetração do corante $(\mathrm{p}<0,01)$.
\end{abstract}

UNITERMOS: Infiltração dentária; Dente decíduo; Restauração dentária permanente.

\section{INTRODUÇÃO}

O cimento de ionômero de vidro (CIV) tem demonstrado ser um material restaurador promissor na prevenção da cárie secundária, pois libera flúor, aumentando a resistência das estruturas dentárias que o circundam ${ }^{11}$. Além dessa característica, possui ainda a capacidade de aderir-se quimicamente aos tecidos duros do dente ${ }^{28}$ e seu coeficiente de expansão térmica linear é semelhante ao da coroa dentária, reduzindo a microinfiltração das restaurações ${ }^{29}$.

Em 1992, surgiram no mercado os primeiros cimentos de ionômero de vidro modificados por resina (CIVMR) restauradores ${ }^{7}$, apresentando propriedades importantes como: biocompatibilidade, boa resistência à compressão, fluidez para injeção na cavidade, variedade de cor e pequena solubilidade nos fluidos bucais além das já citadas anteriormente $^{7}$. Ao contrário dos CIV convencionais que necessitam de sete a dez minutos para a presa ini- cial, as novas fórmulas apresentam duplo mecanismo de endurecimento. O componente resinoso polimeriza em 30 a 60 segundos pela exposição à luz visivel, fornecendo ao cimento um endurecimento inicial satisfatório. E mesmo após a polimerização da resina, a reação ácido-base do CIV continua a se processar normalmente dentro da matriz resinosa ${ }^{8}$.

Testes in vitro de infiltração marginal com os CIVMR desenvolvidos para restauração indicam que os diferentes materiais exibem resultados variáveis nos testes de microinfiltração, sendo que nem todos promovem vedamento marginal superior aos seus correspondentes convencionais ${ }^{23}$.

Vários materiais têm sido colocados no mercado com o apelo de serem CIV fotopolimerizáveis, porém não devem ser classificados como tal, por apresentarem características mais próximas de resina composta. Tais materiais são usualmente chamados de compômeros e podem ser classificados como resinas modificadas por poliácidos.

$\dagger$ Parte da Tese de Doutorado em Odontopediatria apresentada à Faculdade de Odontologia de Bauru da USP.

* Professor Doutor de Odontopediatria da Faculdade de Odontologia da Universidade Sagrado Coração - Bauru.

** Professores Doutores de Odontopediatria da Faculdade de Odontologia de Bauru da USP. 
CARRARA, C. E.; ABDO, R. C. C.; SILVA, S. M. B. da. Avaliação da infiltração marginal de materiais restauradores adesivos em dentes decíduos. Pesqui Odontol Bras, v. 15, n. 2, p. 151-156, abr./jun. 2001.

Tanto os CIVMR como os compômeros têm sido amplamente utilizados em Odontopediatria. Porém, os estudos com estes materiais aplicados sobre os tecidos duros de dentes decíduos, ao contrário dos dentes permanentes, ainda são pouco freqüentes ${ }^{15}$.

Sendo a infiltração marginal ainda um desafio à prática clínica, idealizou-se este trabalho para avaliar, in vitro e em dentes decíduos, a infiltração de alguns materiais restauradores, com seus respectivos sistemas adesivos, comercializados no mercado brasileiro: o CIV convencional, ChelonFil ${ }^{*}$ o CIVMR Vitremer** e o compômero Compoglass $-\mathrm{F}^{* * *}$ e a resina composta $Z 100^{* * * *}$.

\section{MATERIAL E MÉTODOS}

Cavidades classe $\mathrm{V}$ foram confeccionadas na face vestibular de 40 caninos decíduos, um milímetro aquém da junção cemento-esmalte, em esmalte, medindo um milimetro de profundidade, três milimetros no sentido mésio-distal e dois no gêngivo-oclusal. Os dentes foram divididos em quatro grupos de dez dentes cada e restaurados com diferentes materiais, sendo o primeiro restaurado com o CIV convencional Chelon-Fil (grupo I); o segundo com o CIVMR Vitremer (grupo II); o terceiro com a resina composta modificada por poliácidos Compoglass-F (grupo III) e o último com a resina composta Z100 (grupo IV). Os materiais foram manipulados segundo as instruções dos respectivos fabricantes.

Os dentes já restaurados passaram por termociclagem em água deionizada, nas temperaturas de 5 e $55^{\circ} \mathrm{C}$, perfazendo um total de 500 ciclos. O tempo de permanência dos corpos-de-prova em cada banho foi de 15 segundos e o de transição entre um banho e outro foi de 10 segundos.

Após a ciclagem térmica, os dentes foram isolados com duas camadas de esmalte para unha, deixando exposta a restauração e um milimetro ao seu redor. Em seqüência, os espécimes foram imersos em solução de fucsina básica a 0,5\%, onde permaneceram por 24 horas à temperatura de $37^{\circ} \mathrm{C}$. Após a remoção do corante, os dentes foram lavados, decapados do esmalte de unha e seccionados longitudinalmente através das restaura- ções, no sentido vestíbulo-lingual, de modo a obter três fatias para avaliação em microscópio óptico.

A extensão de penetração do corante foi avaliada nas paredes gengival e oclusal empregando-se o seguinte critério de escores: 0 - nenhuma infiltração; 1 - infiltração do corante até a metade da profundidade da parede circundante; 2 - infiltração do corante além da metade da profundidade da parede circundante, porém não atingindo a parede axial; 3 - infiltração do corante atingindo a parede axial ou seu prolongamento.

A segunda avaliação foi realizada com o auxílio de um microscópio óptico de medida, munido de ocular micrometrada de aumento $12,5 \mathrm{X}$ e objetiva $4 \mathrm{X}^{* * * * *}$.

Os dados obtidos foram submetidos ao teste estatístico de Kruskal-Wallis para verificar se os grupos estudados eram ou não diferentes entre si; e de Miller para identificar onde estariam estas diferenças, ambos com nivel de significância estipulado em $1 \%$.

\section{RESULTADOS}

O Gráfico 1 apresenta a infiltração marginal linear média para os diferentes materiais, nas paredes cervical e oclusal.

Os cimentos de ionômero de vidro Chelon-Fil e Vitremer apresentaram uma infiltração maior na parede cervical do que na oclusal, sendo que a diferença foi estatisticamente significante $(p<0,01)$. Os materiais Compoglass-F e Z100 apresentaram infiltração marginal na parede cervical semelhante

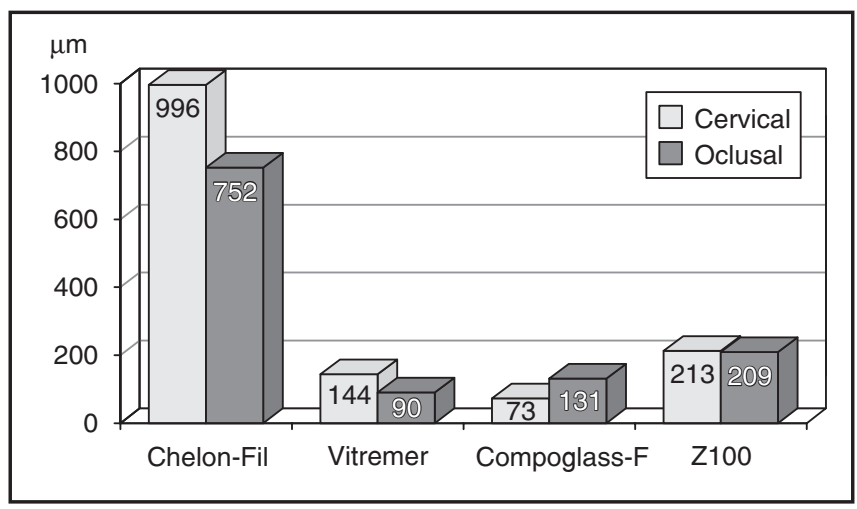

GRÁFICO 1 - Média da infiltração marginal, em micrômetros, nas paredes cervical e oclusal, para os diferentes materiais.

\footnotetext{
*ESPE Dental-Medizin, Seefeld, Alemanha.

**3M/Dental Products, St. Paul, MN, EUA.

*** Ivoclar/Vivadent, Ellvangen, Alemanha.

****3M/Dental Products, St. Paul, MN, EUA.

*****Carl Zeiss, Alemanha.
} 
CARRARA, C. E.; ABDO, R. C. C.; SILVA, S. M. B. da. Avaliação da infiltração marginal de materiais restauradores adesivos em dentes decíduos. Pesqui Odontol Bras, v. 15, n. 2, p. 151-156, abr./jun. 2001.

TABELA 1 - Número de escores de infiltração marginal observado nas paredes cervical e oclusal, para os diferentes materiais.

\begin{tabular}{c|c|c|c|c|c|c|c|c}
\hline \multirow{2}{*}{ Material } & \multicolumn{2}{|c|}{ Chelon-Fil } & \multicolumn{2}{c|}{ Vitremer } & \multicolumn{2}{c|}{ Compoglass-F } & \multicolumn{2}{c}{ Z100 } \\
\cline { 2 - 10 } & cervical & oclusal & cervical & oclusal & cervical & oclusal & cervical & oclusal \\
\hline 0 & 1 & 3 & 12 & 18 & 15 & 18 & 12 & 18 \\
\hline 1 & 7 & 11 & 17 & 11 & 14 & 8 & 14 & 6 \\
\hline 2 & 8 & 6 & 1 & 1 & 0 & 2 & 0 & 0 \\
\hline 3 & 13 & 9 & 0 & 0 & 0 & 1 & 30 \\
\hline \hline
\end{tabular}

à da parede oclusal, não havendo diferença estatisticamente significante.

Comparando-se a infiltração marginal dos diferentes materiais, pode-se observar que não houve diferença significante entre Vitremer, Compoglass-F e Z100, porém os três apresentaram vedamento marginal superior ao CIV convencional Chelon-Fil, tanto na parede oclusal como na gengival.

Os resultados obtidos para a infiltração marginal, medida através de escores (Tabela 1), foram semelhantes aos obtidos através da medida linear. Não houve diferença significante entre os materiais Vitremer, Compoglass-F e Z100, porém os três apresentaram vedamento marginal superior ao do ionômero convencional Chelon-Fil, tanto na parede oclusal como na gengival.

\section{DISCUSSÃO}

Muitos trabalhos de pesquisa ${ }^{9,10,14,17,24}$, também corroborados por este, têm demonstrado que os CIV não são capazes de evitar totalmente a infiltração marginal quando utilizados como restauradores ou bases forradoras. DERBYSHIRE et al. ${ }^{9}$ (1988) compararam a infiltração ao redor de restaurações de resina composta com e sem base de CIV. Esses autores não conseguiram demonstrar diferença entre as duas técnicas restauradoras. Em contrapartida, muitos pesquisadores ${ }^{4,12,16,20,21}$ têm obtido resultados consistentes, mostrando uma redução na microinfiltração com a utilização dos CIV nas margens gengivais.

Desde seu desenvolvimento no início da década de 70 por WILSON; KENT ${ }^{28}$ (1972), os CIV vêm sofrendo muitas modificações na fórmula, resultando em melhor resistência mecânica, aumento na translucidez e, o mais importante, diminuição no tempo de endurecimento ${ }^{29}$. Nos últimos anos, essa evolução na formulação dos CIV levou à intro- dução da versão híbrida do material, a qual é fotopolimerizável. Esse tipo de cimento de ionômero de vidro (CIVMR) foi desenvolvido para ajudar a solucionar os problemas de sensibilidade à umidade e baixa resistência mecânica inicial associadas aos CIV convencionais, procurando preservar suas principais propriedades, como a liberação de flúor e a adesividade às estruturas dentárias ${ }^{5,23}$.

BRACKETT et al. ${ }^{2}$ (1995) estudaram a infiltração marginal dos materiais Fuji II LC e Photac-Fil, dois CIVMR, comparando-os com o Ketac-Fil, um CIV convencional. Não observaram diferença estatisticamente significante entre eles. HALLETT; GARCIA-GODOY $^{13}$ (1993), em estudo semelhante, observaram que, nas margens de esmalte, o Fuji II LC permitia maior infiltração que o convencional, mas na margem de cemento os resultados foram parecidos. VIRMANI et al. $^{26}$ (1997) observaram infiltração menor com CIV convencional Fuji IX que com o Vitremer, o contrário do que observou PIN ${ }^{18}$ (1997). Analisando a formação de "gaps" na interface dente/restauração, SIDHU ${ }^{22}$ (1994) observou uma superioridade dos materiais Variglass e Fuji II LC sobre o convencional Fuji Cap II. Os resultados apresentados por este trabalho mostram que o comportamento do CIV convencional foi pior que o de todos os outros materiais.

YU et al..$^{30}$ (1995) compararam a infiltração marginal de quatro cimentos que liberam flúor, entre eles o Vitremer e o Variglass/Caulk Dentisply. Contrariamente ao trabalho de CARRARA ${ }^{3}$ (1995), que observou uma grande superioridade do CIVMR Vitremer sobre o compômero Variglass, esses autores não observaram diferença entre os materiais no que diz respeito à infiltração marginal. Também estudando infiltração marginal de CIVMR e compômero, SALLES ${ }^{19}$ (1997) obteve resultados superiores com o Vitremer, comparado com o compômero Dyract. No presente trabalho, o CIVMR Vi- 
CARRARA, C. E.; ABDO, R. C. C.; SILVA, S. M. B. da. Avaliação da infiltração marginal de materiais restauradores adesivos em dentes decíduos. Pesqui Odontol Bras, v. 15, n. 2, p. 151-156, abr./jun. 2001.

tremer e o compômero Compoglass-F comportaram-se de maneira semelhante quanto à infiltração marginal.

Os resultados deste trabalho demostram que nenhum dos materiais estudados foi capaz de impedir totalmente a infiltração marginal, porém o CIV convencional Chelon-Fil permitiu penetração do corante maior que os outros, com diferença estatística significante $(\mathrm{p}<0,01)$.

O CIVMR Vitremer e o CIV Chelon-Fil apresentaram infiltração marginal maior na parede cervical que na oclusal. Já os materiais resinosos Compoglass-F e Z100 não apresentaram diferenças quanto às paredes estudadas, provavelmente por se utilizarem do condicionamento do esmalte com ácido fosfórico a $37 \%$ e posterior aplicação do adesivo. Apesar da espessura do esmalte ser bem menor na parede cervical, esta deve ter sido suficiente para permitir uma infiltração marginal semelhante à da parede oclusal.

Não se observaram diferenças na infiltração marginal quando se realizou a medida linear até a parede da câmara pulpar ou quando se atribuíram escores. Com os dados observados no trabalho mostrando uma infiltração do corante muito semelhante para os materiais Vitremer, Compoglass-F e Z100, nunca atingindo a parede de fundo da cavidade e não apresentando penetração nos canalículos dentinários - e o Chelon-Fil apresentando infiltração que quase sempre atingiu a parede de fundo da cavidade, penetrando na dentina em direção à polpa - os resultados não poderiam ser diferentes. Porém, com a análise através de escores ou apenas medindo-se a infiltração linearmente até a parede de fundo da cavidade, corre-se o risco de se considerarem iguais as infiltrações de materiais que vedam a embocadura dos canalículos dentinários com a de materiais que não o fazem, permitindo infiltração através da dentina em direção à polpa.

Pelos testes realizados, observou-se um desempenho muito semelhante entre o CIVMR Vitremer e o compômero Compoglass-F. Porém, estes materiais apresentam outras propriedades que são diferentes e que também devem ser consideradas no momento de sua indicação. Por exemplo, a solubilidade do Vitremer é maior que a do Compoglass-F, em contrapartida, sua liberação de flúor também é maior $^{1,6}$, com capacidade de formar uma camada enriquecida por íons flúor no esmalte adjacente tornando-o mais resistente à desmineralização por ácidos ${ }^{27}$.

A liberação de flúor apresentada pelo Vitremer ${ }^{1,6}$ e sua atividade antimicrobiana ${ }^{6}$ são características bastante desejadas em Odontopediatria, principalmente nos casos de grande atividade de cárie. Este fator associado aos bons resultados nos testes aqui apresentados asseguram sua indicação em dentes decíduos em várias situações.

Por suas propriedades serem mais parecidas com as das resinas compostas, o Compoglass-F pode ser indicado para cavidades mais amplas e em dentes posteriores, alguns autores o indicam para cavidades classe II em dentes decíduos ${ }^{5,25}$. Porém, este material deve ser indicado para pacientes com atividade de cárie não muito intensa.

Outra vantagem com relação ao Compoglass-F é a facilidade de manipulação. Não há necessidade de mistura e espatulação. O material já vem pronto para ser levado à cavidade, acondicionado em porções e em dispositivos que se acoplam a seringas aplicadoras. Estas facilidades de técnica são sempre interessantes, principalmente para o tratamento de crianças.

\section{CONCLUSÕES}

Os resultados obtidos com a metodologia utilizada neste trabalho permitem concluir que:

- nenhum dos materiais estudados foi capaz de impedir a infiltração marginal;

- o material Chelon-Fil permitiu infiltração marginal maior que os outros materiais estudados $(p<0,01)$;

- a infiltração marginal na parede cervical foi maior que na parede oclusal, para os materiais Vitremer e Chelon-Fil ( $p<0,01)$;

- a infiltração marginal foi semelhante nas paredes cervical e oclusal, para os materiais Compoglass-F e Z100 ( $\mathrm{p}<0,01)$;

- não houve diferença entre os resultados obtidos com os dois métodos de avaliação da infiltração marginal, escore e medida linear.

\section{AGRADECIMENTOS}

À CAPES, por ter financiado a pesquisa. 
CARRARA, C. E.; ABDO, R. C. C.; SILVA, S. M. B. da. Avaliação da infiltração marginal de materiais restauradores adesivos em dentes decíduos. Pesqui Odontol Bras, v. 15, n. 2, p. 151-156, abr./jun. 2001.

CARRARA, C. E.; ABDO, R. C. C.; SILVA, S. M. B. da. Evaluation of the microleakage of restorative materials in deciduous teeth. Pesqui Odontol Bras, v. 15, n. 2, p. 151-156, abr./jun. 2001.

The microleakage of Chelon-Fil, Vitremer, Compoglass-F and Z100 was evaluated in deciduous teeth. Class V restorations were placed in the buccal aspect of 40 extracted primary canines; they were thermocycled, and immersed in $0.5 \%$ basic fuchsin. Three slices of each tooth were obtained in order to analyze the penetration of dye in the gingival and occlusal walls, under magnification of $40 \mathrm{X}$. The results showed that Chelon-Fil had the highest degree of marginal leakage. Vitremer, Compoglass-F and Z100 presented similar penetration of dye. The microleakage of Chelon-Fil and Vitremer was greater in the gingival wall than in the occlusal wall. Microleakage was the same in both gingival and occlusal walls when cavities were filled, either with Z100 or with Compoglass-F.

UNITERMS: Dental leakage; Tooth, deciduous; Dental restoration, permanent.

\section{REFERÊNCIAS BIBLIOGRÁFICAS}

1. BERTACCHINI, S. M.; ABATE, P. F.; BLANK, A. et al. Solubility and fluoride release in ionomers and compomers. Quintessence Int, v. 30, n. 3, p. 193-197, Mar. 1999.

2. BRACKETT, W. W.; GILPATRIC, R. O.; GUNNIN, T. D. Microleakage of light-cured glass ionomer restorative materials. J Dent Res, v. 74, n. 3, Mar. 1995. (Resumo n. 766)

3. CARRARA, C. E. Avaliação da infiltração marginal de dois cimentos resinosos restauradores que liberam flúor. Bauru, 1995. 75 p. Dissertação (Mestrado) Faculdade de Odontologia de Bauru, Universidade de São Paulo.

4. COOLEY, R. L.; BARKMEIER, W. W. Dentinal shear bond strength, microleakage, and contraction gap of visible light-polymerized liners/base. Quintessence Int, v. 22, n. 6, p. 467-474, June 1991.

5. CORTÉS, O.; GARCÍA, C.; PÉREZ, L.; BRAVO, L. A. A comparison of the bond strength to enamel and dentin of two compomers: an in vitro study. ASDC J Dent Child, v. 65, n. 1, p. 29-31, Jan./Feb. 1998.

6. COSTA, B. Avaliação in vitro da atividade antimicrobiana e liberação de flúor de cimentos de ionômero de vidro restauradores quimicos e fotoativados. Bauru, 1995. 121 p. Dissertação (Mestrado) - Faculdade de Odontologia de Bauru, Universidade de São Paulo.

7. CROLL, T. P.; KILIAN, C. M. Visible light-hardened glass-ionomer-resin cement restorations for primary teeth: new developments. Quintessence Int, v. 10, n. 10 , p. 679-682, Oct. 1992.

8. CROLL, T. P.; KILLIAN, C. M.; HELPIN. L. A restorative dentistry renaissance for children: light-hardened glass-ionomer/resin cement. ASDC J Dent Child, v. 60, n. 2, p. 89-94, Mar./Apr. 1993.

9. DARBYSHIRE, P. A.; MESSER, L. B.; DOUGLAS, W. H. Microleakage in class II composite restorations bonded to dentin using thermal and load cycling. J Dent Res, v. 67, n. 3 , p. 585-587, Mar. 1988.

10. ERDILEK, N.; OZATA, F.; SEPTCIOGLU, F. Microleakage of glass ionomer cement composit resin and glass ionomer resin cement. J Clin Pediatr Dent, v. 21, n. 4, p. 311-314, 1997.
11. FORSTEN, L. Fluoride release from a glass-ionomer cement. Scand J Dent Res, v. 85, n. 6, p. 503-504, Sept. 1977.

12. FUTATSUKI, M.; NAKATA, M. In vitro marginal leakage of class II composite restorations by thermal cycling. $\mathbf{J}$ Clin Pediat Dent, v. 18, n. 3, p. 191-196, June 1994.

13. HALLETT, K. B.; GARCIA-GODOY, F. Microleakage of resin-modified glass ionomer cement restoration: an in vitro study. Dent Mater, v. 9, n. 5/6, p. 306-311, Sept. 1993.

14. HIRSCHFELD, Z.; FRENKEL, A.; ZYSKIND, D. et al. Marginal leakage of class II glass ionomer-composite resin restorations: an in vitro study. J Prosthet Dent, v. 67, n. 2, p. 148-156, Feb. 1992.

15. KIELBASSA, A. M.; WRBAS, K. T.; HELLWIG, E. Initial tensil bond strength of resin-modified glass ionomers and polyacid-modified resins on perfused primary dentin. ASDC J Dent Child, v. 164, n. 3, p. 183-187, May/July 1997.

16. MATHIS, R. S.; DEWALD, J. P.; MOOD, C. R. et al. Marginal leakage in class $\mathrm{V}$ composite resin restorations with glass ionomer liners in vitro. J Prosthet Dent, v. 63, n. 5, p. 522-525, May 1990.

17. MOUNT, G. J.; PAPAGEORGIOU, A.; MAKINSON, O. F. Microleakage in the sandwich technique. Am J Dent, v. 5, n. 4, p. 195-198, Aug. 1992.

18. PIN, M. L. G. Avaliação da microinfiltração marginal em cavidades classe II modificadas, restauradas com cimento de ionômero de vidro, convencional, modificado por resina e com uma resina composta modificada por poliácidos: um estudo in vitro. Bauru, 1997. 124 p. Dissertação (Mestrado) - Faculdade de Odontologia de Bauru, Universidade de São Paulo.

19. SALLES, V. Avaliação in vitro da infiltração marginal de restaurações realizadas com um cimento de ionômero de vidro modificado por resina e uma resina composta modificada por poliácidos associadas a dois sistemas adesivos. Bauru, 1997. 130 p. Dissertação (Mestrado) - Faculdade de Odontologia de Bauru, Universidade de São Paulo.

20. SCHWARTZ, J. L.; ANDERSON, M. H.; PELLEU Jr., G. B. Reducing microleakage with the glass-ionomer/resin sandwich technique. Oper Dent, v. 15, n. 5, p. 186-192, Sept./Oct. 1990. 
CARRARA, C. E.; ABDO, R. C. C.; SILVA, S. M. B. da. Avaliação da infiltração marginal de materiais restauradores adesivos em dentes decíduos. Pesqui Odontol Bras, v. 15, n. 2, p. 151-156, abr./jun. 2001.

21. SHORTALL, A. C. In vitro microleakage of class V composite resin restorations with and without light-cured glass-ionomer (poly alkenoate) cement lining. Eur J Prosthodont Restor Dent, v. 1, n. 1, p. 7-11, Sept. 1992.

22. SIDHU, S. K. Marginal contraction gap formation of light-cured glass ionomers. Am J Dent, v. 7, n. 2, p. 115-118, Apr. 1994.

23. SIDHU, S. K.; WATSON, T. F. Resin-modified glass ionomer materials - a status report for the American Journal of Dentistry. Am J Dent, v. 8, n. 1, p. 59-67, Feb. 1995.

24. SILVA E SOUZA Jr., M. H.; VIEIRA, L. C. C.; NAVARRO, M. F. L. et al. Avaliação da infiltração marginal em esmalte e cemento em cavidades classe II MOD restauradas com cinco sistemas adesivos. Rev Odontol Univ São Paulo, v. 5, n. 2, p. 140-145, jul./dez. 1991.

25. TUlunOGLU, O.; AYHAN H.; OLMZ A. et al. The effect of cavity disinfectants on microleakage in dentin-bonding systems. J Clin Pediatr Dent, v. 22, n. 4, p. 299-305, 1998.
26. VAIKUNTAM, J. Resin-modified glass ionomer cements (RMGICs): implications for use in pediatric dentistry. ASDC J Dent Child, v. 164, n. 2, p. 131-134, Mar./Apr. 1997.

27. WALLS, A. W. G.; Mc CABE, J. F.; MURRAY, J. J. Factors influencing the bonding strength between glass polyalkenoate (ionomer) cements and dentin. J Oral Rehabil, v. 15, n. 6, p. 537-547, Nov. 1988.

28. WILSON, A. D.; KENT, B. E. A new translucent cement for dentistry: the glass ionomer cement. Br Dent J, v. 132, n. 4, p. 133-135, Feb.1972.

29. YAP, U. J.; STOKES, A. N.; PEARSON, G. J. Concepts of adhesion: a review. New Zealand Dent J, v. 90, n. 401, p. 92-98, Sept. 1994.

30. YU, X. Y.; WIECZKOWSKI, G.; DAVIS, E. et al. Shear bond strength and microleakage of four hybrid glass ionomer resin systems. J Dent Res, v. 74, n. 3, Mar. 1995. (Resumo n. 764) 